Sociologie et sociétés

\title{
Le risque de cancer du sein en France : un mode unique de prévention
}

\section{The risk of breast cancer in France : a single method of prevention}

\section{Marie Ménoret}

Volume 39, numéro 1, printemps 2007

Risque et santé

Risk and health

URI : https://id.erudit.org/iderudit/016936ar

DOI : https://doi.org/10.7202/016936ar

Aller au sommaire du numéro

Éditeur(s)

Les Presses de l'Université de Montréal

ISSN

0038-030X (imprimé)

1492-1375 (numérique)

Découvrir la revue

Citer cet article

Ménoret, M. (2007). Le risque de cancer du sein en France : un mode unique de prévention. Sociologie et sociétés, 39(1), 145-160.

https://doi.org/10.7202/016936ar
Résumé de l'article

Cet article porte sur l'élaboration du risque de cancer du sein en France et sur ses conséquences sociales. Il s'appuie sur des données historiques et sociologiques pour tracer l'évolution de sa prévention, de la fin du XIX ${ }^{\mathrm{e}}$ siècle aux tests contemporains de prédisposition génétique. Il montre que la détection précoce s'est imposée comme la technique d'excellence de prévention du cancer du sein, dans un monde où les thérapeutiques évoluent peu en matière de guérison, d'une part, et dans lequel les discours alternatifs n'ont eu que très peu d'audience auprès des publics concernés, d'autre part. Basée exclusivement sur le dépistage, l'entreprise française de prévention du cancer du sein a progressivement transformé des personnes en bonne santé en patientes asymptomatiques - dans un processus de médicalisation du risque - et des populations ciblées de femmes en population à risque - dans un processus de naturalisation du même risque. L'entreprise de surveillance qui organise cette situation est l'oeuvre d'une autonomie médicale qui légitime un " faute de mieux », en l'absence de remède efficace. 


\section{Le risque de cancer du sein en France: un mode unique de prévention}

\section{MARIE MÉ NORET}

Département de sociologie

Université Paris 8

2 , rue de la Liberté

93526 Saint-Denis Cedex, France

Courriel : menoret@vjf.cnrs.fr

U

N FACTeur de Risque, dans le Registre de la prévention médicale, c'est quelque chose qui accroît la probabilité d'avoir une maladie. Dans le domaine du cancer, quelques-uns de ces facteurs ne peuvent être changés, comme la couleur de la peau ou bien l'âge, voire encore le sexe. Certains d'entre eux, au contraire, peuvent être modifiés en vue d'une amélioration du niveau de santé des personnes. Parmi ceux-ci, on en repère qui ont trait à l'environnement — l'amiante ou les pesticides, par exemple, dont les effets délétères sont documentés depuis des décennies - et d'autres qui sont liés à des choix plus individuels — tabac, alcool, exposition sans précaution au soleil, etc. bref: à des comportements. Cette triple contingence induit différents niveaux de discours et différentes actions en matière de santé publique. Globalement, ces discours et ces actions se déclinent en trois grandes perspectives. La première s'emploie à sensibiliser des groupes sociaux déterminés et les engage à une forme d'autosurveillance. La deuxième vise à impulser une politique environnementale afin de minimiser des risques exogènes. La troisième perspective prend les individus pour cible et les invite à adopter des attitudes visant à neutraliser personnellement les dangers qui les guettent. En France, où chaque année 12000 femmes, tous âges confondus, meurent d'un cancer du sein et 46000 nouveaux cas sont diagnostiqués, la prévention de cette maladie adhère à la première et à la dernière de ces perspectives. Pour prévenir l'apparition de maux au demeurant 
imprédictibles, on évalue l'incalculable afin que de possibles futurs événements deviennent l'objet d'une action au présent. En médecine comme ailleurs, la notion de risque est une affaire de recensement et d'évaluation qui tente l'improbable alliance cognitive entre collectif et individuel. Le résultat en est cette moyenne statistique sur laquelle le risque est fondé et dans laquelle l'individu se trouve le plus souvent dissous (Hacking, 1975 et 1990; Cassedy, 1984; Rosser Matthews, 1995). En 2004, afin d'améliorer la prévention du cancer du sein, les pouvoirs publics français ont généralisé un dépistage de masse envers une population de femmes ciblée entre 50 et 74 ans. Ainsi, le résultat du calcul du risque du cancer du sein a-t-il simultanément médicalisé et essentialisé, à travers un sexe et une classe d'âge un nouveau champ de responsabilité.

Alors que les recherches en sciences sociales consacrées à cette pathologie suscitent, particulièrement depuis les années 1990, un certain intérêt dans les pays anglo-saxons elles n'ont, pour l'instant, qu'assez peu mobilisé les chercheurs français dans ce mouvement que Lock et Kaufert croient pouvoir appeler une «tradition euro-américaine» de travaux féministes consacrés à la santé des femmes (Lock et Kaufert, 1998: 11). Nos collègues nord-américains sont incontestablement les plus présents sur ce terrain et ils et elles nous proposent une production pluridisciplinaire riche. Des points de vue nombreux: sociohistoriques (Leopold, 1999; Proctor, 1995), politiques (Altman, 1996; Stabiner, 1997), professionnels (Love, 1990; Lerner, 2001), anthropologiques (Kaufert, 1998), sociologiques et philosophiques (Kasper et Ferguson, 2000; Pott, 2000) sont mobilisés pour explorer non seulement les contextes sociaux du cancer du sein, mais également les mystifications dont les femmes sont parfois l'objet à travers les prises en charge qui leur sont réservées. L'absence singulière de la recherche sociologique française dans ce domaine précis rejoint finalement la conjoncture problématique dans laquelle s'inscrit l'analyse proposée ici: à savoir l'indigence remarquable, en France, de point de vue critique collectif de la part des femmes par rapport à la médecine alors même qu'elles en sont toujours les plus fidèles auxiliaires de soins domestiques.

Pour mieux comprendre comment cette situation s'est ancrée dans l'espace social, on a choisi de l'examiner à travers l'exemple de la prise en charge du risque de cancer du sein. L'article est organisé, dans cet objectif, autour des trois axes de réflexion. Le premier observe l'élaboration de la notion même de risque pour le cancer en général, puis celui du sein en particulier; le second examine les logiques techniques ainsi que les relations sociales et économiques qui légitiment les dispositifs émergents de dépistage; un troisième niveau, enfin, s'intéresse aux conséquences de l'émergence de ces normes sur les femmes elles-mêmes. En explorant l'élaboration de ce phénomène du risque auquel sont particulièrement soumises les femmes affiliées à l'institution de prévention du cancer du sein, cet article prolonge mes premiers travaux sur le thème de la cancérologie (Ménoret, 1999). En s'imposant comme la technique d'excellence de prévention du cancer du sein, dans un monde où, d'une part, les thérapeutiques évoluent, mais sans jamais garantir un objectif de guérison et dans lequel, d'autre part, les discours alternatifs — notamment environnementaux — ont peu d'audience auprès des experts impliqués dans l'entreprise de surveillance, le dépistage, en médicalisant un 
secteur important de santé publique, transforme progressivement des femmes en bonne santé en des populations entières à risque.

\section{LES FONDEMENTS DU RISQUE DE CANCER}

Prévenir pour ne pas avoir à guérir : si la cause de la prévention médicale est entendue, elle s'impose plus particulièrement devant des pathologies qui résistent aux objectifs curatifs de la médecine. Or, la guérison reste encore quasiment une fois sur deux inaccessible, tous cancers confondus, dans la médecine occidentale contemporaine. Cette situation n'est pas nouvelle: elle s'est imposée socialement comme une donnée particulièrement problématique depuis que le cancer est devenu une des causes majeures de mortalité. C'est la maitrise de la mortalité infectieuse opérée dans le courant du XIX ${ }^{\mathrm{e}}$ siècle qui va permettre aux cancers d'accéder au statut de cause majeure de la mort. Le docteur Jacques Bertillon, qui crée à cette époque la première classification des maladies permettant d'effectuer des relevés épidémiologiques d'entités cliniques bien définies, constate dès 1906 que «le cancer a fait une percée spectaculaire » puisqu’il donne 5,5\% des morts à Paris vers 1895. En 1923, Jean Bergonié, promoteur du futur centre anticancéreux français, justifie d'ailleurs son projet d' « usine à guérir» en insistant sur le « déchet social» que constitue le malade cancéreux sans espoir de guérison (Pinell, 1992). Au sein du futur dispositif, la prévention est déjà un instrument essentiel de l'entreprise de soins tandis que, parallèlement, la notion de risque émerge dans une épistémologie d'épidémiologistes qui limite déjà les facteurs dits environnementaux à des comportements individuels.

Le cancer broncho-pulmonaire a été le premier à retenir l'attention de ces nouveaux experts après la Première Guerre mondiale et à documenter les intuitions que certains cliniciens, notamment des chirurgiens thoraciques, énonçaient dès 1912 sur le rôle que la fumée du tabac pourrait jouer dans l'étiologie du cancer broncho-pulmonaire (Lickint, 1935). La plus importante des premières grandes enquêtes épidémiologiques va être réalisée en Grande-Bretagne de 1948 à 1952 par Doll et Hill (Schwartz, 1994). Le rôle imputé au tabac dans l'apparition d'un cancer broncho-pulmonaire s'y trouve fortement souligné mais, dans un premier temps au moins, ces résultats vont être assez critiqués sur des questions d'ordre méthodologique. Doll et Peto poursuivent leurs recherches dans ce domaine, spécialement dans une grande enquête auprès de médecins anglais, dont les habitudes tabagiques seront suivies pendant 20 ans et mises en relation avec l'apparition, ou non, de cancer bronchique. Les conclusions radicales de ces auteurs, selon lesquelles le tabac se trouve être la cause principale et directe du cancer, vont encore une fois être très discutées (Fisher, 1957; Yerushalmy et Palmer, 1959; Doll et Peto, 1976). Toutes ces controverses contribuent largement à affiner la notion de risque qui se trouve au cœur de ces débats multiples. Fagot-Largeault écrit:

N'est-il pas abusif d'appeler «cause» un facteur auquel seul un risque, ou plutôt une fraction d'un risque est attribuable? Et plus abusif encore de désigner la fumée du tabac comme «la cause» du cancer broncho-pulmonaire, quand il s'agit d'un fragment de cause? Doll et Peto s'en expliquent [...] : le tabac est le seul facteur identifié auquel soit assignable 
la surmortalité des fumeurs par cancer broncho-pulmonaire, et il rend compte de toute cette surmortalité (sans compter une surmortalité par d'autres cancers et par maladies cardio-vasculaires). Un facteur qui influence la part «évitable» du risque, c'est une cause (efficace); un facteur responsable de toute la part évitable du risque, c'est la cause (efficace). (Fagot-Largeault, 1989:161)

Outre l'affinement des méthodes liées à l'usage de la statistique, ces polémiques ont également pour conséquence de problématiser leur interprétation (Cuyler Hammond et Horn, 1958). À l'opposé du pôle prévenir, celui du guérir fait également débat. Est-il possible qu'un cancer soit curable, guérissable? Cette question aiguë, Comment guérir un cancer?, fait l'objet de quelques spéculations depuis plusieurs décennies déjà. L'une d'entre elles, qui concerne particulièrement le cancer du sein, va connaître une large diffusion. Fin XIX ${ }^{\mathrm{e}}$, un chirurgien, William Halsted, établit une théorie censée faire passer le cancer du sein de l'état de maladie mortelle à celui de maladie curable (Lerner, 2001). En s'appuyant sur le fait que ce type de cancer est d'abord une maladie localisée, puis qu'il s'étend ensuite de façon graduelle vers les tissus voisins, Halsted met au point une procédure chirurgicale qui consiste à enlever autant de tissu potentiellement cancéreux que possible. Cette mastectomie élargie implique non seulement l'ablation du sein, mais également celle des muscles pectoraux qui recouvrent les côtes. Halsted va s'employer à démontrer que cette procédure chirurgicale peut guérir de manière permanente des cancers du sein détectés assez tôt. Le postulat qui se met en place à la faveur de cette innovation c'est, comme l'observe Lerner, d'attaquer vite et fort.

La technique d'Halsted va représenter pendant près d'un siècle l'intervention de référence, avant d'être remplacée par l'intervention dite de Patay qui conserve les muscles pectoraux. Le second thème du postulat vite et fort va tomber lentement en discrédit, en tout cas dans sa forme chirurgicale dominante extrêmement invasive. Celle-ci rencontrera, de fait, deux critiques essentielles (Montini et Ruzeck, 1989). La première à la fin des années 1940 : des études épidémiologiques montrent que la mastectomie radicale ne change rien au pronostic. La seconde, trente ans plus tard, quand l'expansion des concepts du féminisme américain et l'émergence d'une revendication en termes de droits des patients amènent des femmes avec un cancer du sein à exiger non seulement des opérations moins mutilantes, mais également à revendiquer leur pleine participation au processus de décision médicale. À côté de l'argumentaire épidémiologique et statistique, c'est cette remise en cause de l'autorité médicale qui vainc finalement l'hégémonie de la mastectomie radicale.

Le premier thème du principe relatif à la précocité de l'intervention: attaquer vite reste, lui, toujours de rigueur. L'American Cancer Society (ACS), comme la Ligue nationale contre le cancer (LNCC) en France, militent pour la détection précoce des cancers depuis le début du $\mathrm{xx}^{\mathrm{e}}$ siècle. Cette injonction collective s'élabore tout d'abord sur un discours qui préconise de ne pas tarder à consulter un médecin si des signes visibles de cancer du sein sont observés. Puis, au début des années 1970, les pratiques de détection précoce se déplacent, à travers la recherche de signes invisibles, vers des populations asymptomatiques. Avec l'avènement de la mammographie, le fait d'être 
une femme signifie que l'on s'élève au rang de population à risque. En devenant de potentiels sujets de l'appareil de surveillance et de détection du cancer du sein, les femmes sont invitées à participer de manière plus ou moins active à ce mouvement global.

\section{SURVEILLER, DIAG NOSTIQUER, DÉPISTER}

Dans le courant du $\mathrm{xx}^{\mathrm{e}}$ siècle, la surveillance des femmes occidentales s'est développée en trois étapes distinctes. Afin de prévenir le cancer du sein, les médecins occidentaux ont, à la fin des années 1930, mis au point une technique de surveillance clinique, exercée donc dans le cabinet du professionnel à l'occasion d'une consultation médicale, fondée sur un examen visuel et sur la palpation, par l'expert, de nodules supposés suspects. Cette technique est alors prônée afin d'aider le diagnostic des médecins avant que le cancer n'ait atteint des stades trop développés et situe donc l'objet de surveillance dans la recherche de signes cliniques. La seconde technique de surveillance systématisée est l'autopalpation. Celle-ci sort du cabinet du médecin et concerne donc, cette fois, directement les femmes. Alors qu'elle reste peu préconisée en France où le corps médical partage fort peu sa compétence avec les profanes, l'American Cancer Society en fait, en revanche, une promotion assidue. À la fin des années 1940, l'ACS produit par exemple un petit film documentaire destiné à enseigner à toutes les femmes comment examiner leur seins afin de rechercher des signes éventuels de cancer (Kevles, 1997). Entre 1949 et 1953, plus de 600 copies de ce film vont être distribuées à travers les États-Unis et plus de 3 millions de femmes participeront ainsi à ces séances en faveur du dépistage sponsorisées par l'American Cancer Society. Vingt ans plus tard, les examens cliniques des seins et l'autopalpation sont rejoints par une troisième technique de dépistage, la mammographie, qui va être intégrée dans un système de grande diffusion. À partir des années 1970, cette dernière technique est supposée être un outil fiable de dépistage des populations asymptomatiques. Elle devient, dans les décennies qui suivent, une pratique médicale de plus en plus courante. Fait remarquable et remarqué (Boissonnat, 2003), c'est sans la moindre concertation publique ni politique que se diffuse, dans une France dont l'État se révèle souvent bienveillant envers l'autonomie de son corps médical (Peneff, 2005), cette procédure de surveillance qui inscrit véritablement le cancer du sein dans le monde du risque et, partant, son support essentiel : les femmes. Les gynécologues, les industriels et les praticiens libéraux, notamment radiologues, seront les maîtres d'œuvre de cette diffusion qui s'impose aisément aux autorités sanitaires qui n’ont plus qu'à en entériner le principe.

L'histoire de cette diffusion prend encore quelque temps avant que, dans les années 1980, la mammographie achève de s'imposer comme un élément essentiel du dispositif de surveillance en France. La première unité radiologique explicitement dédiée à l'imagerie du sein avait été mise sur pied en 1951, mais les machines consacrées à la mammographie, c'est-à-dire utilisées spécialement pour les seins n'arrivent pas sur le marché avant 1967, car un segment professionnel, très présent dans les prises en charge du cancer du sein, résiste. Les chirurgiens ont encore une place décisive à cette époque 
en cancérologie. Or, ils considèrent cette technologie comme superflue à l'égard de leur propre travail diagnostique. Ils lui préferent la biopsie, plus invasive mais bien plus performante, selon eux, pour analyser une lésion suspecte, que des appareils à photographier l'intérieur des seins.

Jusque dans les années 1980, en France, la pratique de la mammographie évolue donc en restant essentiellement cette activité à but diagnostique boudée par les chirurgiens. La proportion d'examens radiologiques réalisés à titre systématique dans les cabinets de radiologie français n'atteint pas $9 \%$ en 1982, la moitié d'entre eux correspondant en outre à des clichés pulmonaires (Fagnani et al., 1985). Le dépistage reste faible, les innovateurs demeurent dans une tradition clinique et la notion de risque est en panne puisque le mammographe est une machine à diagnostiquer et non une machine à dépister. Sa mutation préventive va venir d'Europe du Nord.

Les choses sont en effet différentes en Suède où, dès le milieu des années 1970, une évaluation du dépistage du cancer du sein par mammographie avait été entreprise auprès de la population générale. À peine publiés, les résultats obtenus sont largement diffusés et s'imposent comme une évidence, tant dans la presse médicale que dans la presse féminine (Tabar et al., 1985). À la fin des années 1980, le dépistage du cancer du sein se généralise donc aussi dans l'Hexagone. Bien que sa capacité à réduire la mortalité ne soit pas formellement établie, la mammographie se propage en médecine de ville, avant même la publication d'un rapport officiel préconisant la généralisation de la mammographie de dépistage (Cachin, 1986). Aucune recommandation systématique n'accompagnera ce développement en France avant qu'une conférence de consensus soit consacrée à ce thème en 1989. Parallèlement, des recommandations seront bientôt formulées au niveau européen (European Group for Breast Cancer Screening, 1990).

On observe dès lors une très forte progression des mammographies pratiquées dans les cabinets médicaux qui indique que le dépistage commence à se trouver une clientèle. L'émergence d'une conviction partagée par les femmes de se faire dépister est notamment à inscrire au bénéfice de l'action de la Ligue nationale contre le cancer en France. Mais le nouveau segment professionnel qui vient de se constituer autour de la pathologie mammaire, la sénologie, qui regroupe des radiologues et des gynécologues, joue également un rôle majeur dans la diffusion du dépistage mammographique (Lefaure et al., 1990; Le Gales et al., 1990; Wait et al., 1997). Le risque qui est pris en charge dès le début de l'entreprise de surveillance mammographique est celui d'individus et non de groupes déterminés par un risque épidémiologique avéré de population ciblée. Le modèle préventif qui se met en place n'est pas celui d'un dépistage populationnel, mais relève d'une démarche libérale qui cible les femmes en général.

Le dépistage des populations en bonne santé s'accélère donc, en France, à partir du milieu des années 1980, sur un mode individuel pratiqué en secteur libéral et non sur un principe organisé de surveillance de masse. Les discours incitatifs sont exclusivement des discours de médecins libéraux, à la différence des États-Unis par exemple, où ces discours sont reproduits par des groupes de pression constitués de femmes représentant tendanciellement les classes moyennes instruites, blanches et sans problèmes particuliers 
d'accès aux soins. Le développement le plus important dans la diffusion du dépistage mammographique, en dehors des campagnes soutenues de l'American Cancer Society d'un côté de l'Atlantique et de la Ligue nationale contre le cancer de l'autre, arrive avec la fondation du Breast Cancer Awareness Month. Cette initiative américaine, c'est le mois d'octobre dédié au cancer du sein. En 1984, lors de sa première édition, elle est soutenue par de nombreuses organisations professionnelles, gouvernementales, médicales, associatives et financée par l'entreprise Zeneca Pharmaceutical, producteur du tamoxifène, médicament contre le cancer du sein le plus vendu au monde (Paulsen, 1994). Chaque mois d'octobre suivant, à la suite de cette initiative, les pays occidentoeuropéens tentent d'organiser une campagne de publicité dans les médias, avec le relais essentiel de magazines féminins, afin de promouvoir le dépistage mammographique et la détection précoce. La France, là encore, s’y met tardivement, mais quelques associations comme Europa Donna Forum France se lancent à la fin des années 1990 dans la réalisation de ces manifestations annuelles. Deux magazines pour les femmes, MarieFrance d'abord, puis Marie-Claire, s'investissent dans ce mouvement, des femmes de la rédaction souhaitant faire de leur expérience personnelle du cancer du sein une cause publique. Des courses pédestres dédiées à la lutte contre le cancer du sein et exclusivement féminines sont organisées. Parallèlement, des comités féminins pour le dépistage des cancers régionaux sont créés et s'organisent en une fédération nationale. Cette fédération obtient du ministre de la santé en activité, le 8 mars 2005 - Journée de la femme faut-il le rappeler - l'instauration d'une journée nationale du dépistage organisé du cancer du sein. La date retenue est celle du $1^{\text {er }}$ octobre, afin d'ouvrir le mois du cancer du sein.

C'est dans tout ce processus complexe qui vient d'être décrit que s'inscrit l'histoire du risque pour ces femmes, depuis la chirurgie la plus invasive jusqu'à la mammographie. Cette histoire c'est, d'une part, celle d'une traque du plus petit cancer possible — histoire partagée par la plupart des pays occidentaux — mais plus particulièrement pour la France et d'autre part, c'est aussi l'histoire d'une décision publique qui entérine un mouvement médical relayé par des associations chaperonnées par des cancérologues présents dans tous les conseils scientifiques de ces collectifs.

\section{L'INCERTITUDE MÉDICALE AU CGUR DES FIGURES CONTEM PORAINES DU RISQUE}

Dans le processus séculaire de la course effrénée contre le plus petit cancer possible, la doctrine du diagnostic précoce auquel on soumet des populations de gens bien portants depuis le début du $\mathrm{xx}^{\mathrm{e}}$ siècle trouve son apogée avec la découverte des gènes de prédisposition. En effet, depuis le milieu des années 1990, le dépistage génétique, pour l'heure organisé en France à un seul niveau de recherche, vient renforcer ce dispositif. Les gènes de prédisposition au cancer du sein renouvellent peut-être le regard des préventologues sur la maladie mais, finalement, ne font qu'accentuer le principe du toujours plus vite et encore plus précoce. Le cancer est depuis longtemps connu pour être une maladie qui présente parfois des caractères héréditaires. Dès la fin du XIX ${ }^{\mathrm{e}}$ siècle, des enquêtes montrent qu'il existe des familles à cancer et dès 1946, dans le cadre de 
l'Enquête permanente Cancer, des recherches familiales poussées sont entreprises dans ce domaine, qui précisent ces données (Ménoret, 2002). Aucune clinique n'est toutefois envisagée à partir de ces connaissances et il va falloir attendre les années 1990 pour que ce principe soit étayé à l'égard de l'hérédité soupçonnée de certains cancers. À partir de 1994, avec le clonage du premier gène de prédisposition BRCA, l'analyse biologique est devenue un outil de pronostic et le clinicien-chercheur va bientôt pouvoir s'adresser à des sujets non atteints afin de leur proposer un test. Le médecin qui reste le point de passage obligé entre les familles concernées et la recherche fondamentale n'avait, jusqu'alors, rien à proposer à ces personnes, mais à partir de cette date tout change puisque l'on peut cette fois procurer le service du test de prédisposition à leurs membres non atteints de cancer et en cas de résultat positif, accessoirement, des options de traitement de cette nouvelle situation de risque.

Avec les gènes de prédisposition, on trouve pour la première fois dans l'histoire du risque de cancer du sein une mesure préventive stricto sensu. La découverte d'un de ces gènes chez une personne entraîne en effet soit une surveillance médicale soutenue à une fréquence bi-annuelle soit, de manière beaucoup plus rare mais néanmoins attestée de plus en plus fréquemment, une mastectomie prophylactique. On sait, avec Armstrong (1995), que la médecine de surveillance implique la dissolution des catégories distinctes de maladie et de bonne santé et qu'une condition majeure de la prolifération de la prévention médicale consiste à reproblématiser le normal et la maladie elle-même. Ceci est particulièrement vrai pour le cancer du sein dans la mesure où, en dehors de l'ablation prophylactique, il n'existe pas en France, pour les femmes, de prévention qui permette d'éviter son apparition. À techniques et savoirs égaux, les pratiques médicales peuvent en effet différer radicalement en fonction d'évaluations nationales discordantes. Dans certains pays, il est possible de recourir à l'usage de médicaments afin d'empêcher l'apparition d'un cancer du sein. En France, cette possibilité n'est pas offerte dans ce cas. Outre des considérations d'ordre économique, le plus souvent primordiales, de fait, l'exercice médical est aussi largement déterminé par son propre rapport à l'incertitude (Fox, 1980). Et dans le domaine qui nous intéresse, la chimio-prévention l'exemplifie particulièrement puisque le fait d'utiliser des médicaments à titre préventif constitue une ressource disponible dans certains pays occidentaux, mais pas dans tous, pour cause de points de vue divergents quant à l'efficacité des pratiques.

Prenons l'exemple du tamoxifène, connu à l'usage sous le nom de $\operatorname{Nolvadex}^{(\mathrm{r})}$, qui est un anti-œstrogène utilisé depuis longtemps à titre curatif pour traiter les femmes souffrant de certaines formes de cancer du sein. Aux États Unis, depuis 1999, à l'issue d'un essai clinique controversé, ce médicament peut désormais être prescrit de manière préventive aux femmes asymptomatiques présentant un risque élevé de développer un cancer du sein, afin de le réduire au moins à court terme. Les risques encourus par la prise de cette molécule sont loin d'être nuls et justifient la réserve attentiste adoptée notamment par les cancérologues français. Plus récemment, une étude comparant les bénéfices respectifs du tamoxifene et du raloxifene, produit par la firme Eli Lilly and Co 
sous le nom d'Evista ${ }^{(\mathrm{r})}$, concluait sur l'équivalente efficacité du raloxifène par rapport au tamoxifène dans la prévention du cancer du sein. Cette étude, appelée STAR (pour Study of Tamoxifen and Raloxifene), a été menée auprès de 19747 femmes ménopausées et connues - sur la base du Gail Model (Fosket, 2004) — pour présenter un risque de cancer du sein plus élevé que la moyenne. Elle montrait également que le raloxifène, précisément, présente l'avantage d'induire moins d'effets secondaires que le tamoxifene.

Depuis plus d'un siècle, tant les experts du cancer du sein que leurs institutions officielles adhèrent au principe du diagnostic et du traitement précoces qui organise les normes médico-centrées du dépistage, mais cette hégémonie est de plus en plus contestée (Junod et Massé, 2003). L'épidémiologie a montré, par exemple, que la mortalité par cancer du sein était restée stable de 1980 à 1999 chez les femmes de 40 à 79 ans et que le niveau d'extension de l'intervention chirurgicale ou de la radiothérapie n'améliorait pas plus la survie que d'ailleurs la précocité du traitement d'un cancer du sein létal. À ce sombre constat, peu répandu, répond une autre perception, aux antipodes de ces données. Cette autre appréciation, plus largement diffusée et qui fonde implicitement la légitimité du dépistage, est que plus on recherche de cancers du sein, plus on en trouve; que plus on en trouve, plus on en guérit ou, à tout le moins, que plus longue est la durée qui s'écoule entre le diagnostic et le décès.

Entre ces deux perceptions, l'une est sans doute plus fondée que l'autre, mais dans un domaine, celui de l'action politique, où l'evidence based medicine n'est manifestement pas sollicitée (Marks, 1999), le bénéfice lié à la précocité et à l'intensification du dépistage reste visiblement à prouver. Conviction n'est pas confirmation et c'est dans ce contexte qu'un certain nombre de voix, qui n'ont pas renoncé à l'administration de la preuve, s'élèvent aujourd'hui pour exprimer leurs doutes: activistes, associations, épidémiologistes, et même anciens décideurs de santé publique (Welch, 2005; Dab, 2006).

Que sait-on réellement des risques de cancer du sein? Si l'on observe ceux qui organisent le dispositif de prévention et de dépistage, force est de constater que deux facteurs essentiels — à double titre sémantique — sont pris en compte : d'abord le fait d'être une femme et ensuite d'avoir entre 50 ans et 74 ans. En réalité, si l'on considère que le second, celui de l'âge, n'est mobilisé que pour des raisons de fiabilité technique, les experts considèrent en effet que les faux diagnostics sont trop importants avant 50 ans, le seul risque pris en compte à travers les politiques publiques est donc celui d'avoir des seins. La science contemporaine n'a guère produit de savoirs qui permettraient avec certitude de déterminer les causes du cancer du sein. À un double niveau étiologique et démographique, le cancer du sein reste une énigme. La prévention en fonction des facteurs de risque identifiés - âge, précocité des premières règles, nombre de grossesses, gènes de prédisposition par exemple — ne semble pas susceptible d'enrayer son évolution de manière significative. Les risques repérés n'étant pas modifiables, aucune stratégie n'étant scientifiquement validée pour prévenir l'intrusion d'un cancer du sein dans l'existence des personnes, la solvabilité du dispositif de dépistage pourrait être débattue publiquement. Un certain nombre de questions, d'ordre autant épistémologique que 
politique, méritent d'être posées devant un dispositif de prévention, quel qu'il soit. Chaque programme de dépistage est susceptible de faire débat et, tant pour l'expert que pour le profane, les questions peuvent être nombreuses en termes de précision ou en termes de sécurité par exemple. Combien de personnes risquent d'être des fauxnégatifs? De quel type de traitement efficace disposons-nous afin d'éradiquer les symptômes de la maladie dépistée? Ces traitements sont-ils eux-mêmes sans risque? Si le traitement semble être à risque, dans quelle mesure doit-on, peut-on, l'imposer? À des personnes qui peut-être n'auraient jamais développé le moindre symptôme? L'examen de ces questions est aujourd'hui réservé au jugement des experts alors que, comme le soulignait Eliot Freidson dès 1970 dans son examen de la profession médicale, les conséquences morales de choix sociaux inhérents à l'application de savoirs ne sont pas simplement techniques et ne peuvent être déterminés par les seuls experts (Freidson, $1984: 347)$.

Plus près de nous, l'analyse récente des crises sanitaires, écologiques (Chateaureynaud et Torny, 1999), ou celle des politiques publiques du sida (Paicheler, 2002) nous renseignent aussi sur le caractère essentiel de la dichotomie entre la gestion des risques, d'une part, et leur évaluation, d'autre part. Les agences créées ces dernières décennies pour la sécurité — aliments, médicaments notamment — répondaient à cette tension entre l'évaluation du risque par les experts et sa gestion par les politiques, leur fonctionnement illustrant souvent également l'absence du public dans les instances de coordination. En France, à la différence du médicament, la diffusion des tests de dépistage ne fait pas l'objet d'une autorisation de mise sur le marché et tant les experts que les politiques naviguent sur le principe sécuritaire suivant: surveillons les femmes et nous maîtriserons mieux le cancer qui les menace. Dans un contexte d'incertitude tellement imprécis, pourquoi ne pas prendre en compte l'ignorance des experts participant à la décision publique plutôt que leurs connaissances, puisque celles-ci sont loin d'être stabilisées? John Dewey, dans une approche pragmatique de la décision politique et de la détermination des intérêts communs, invitait dès 1927 à l'expérimentation du public contre tout absolutisme d'expert. La France résiste depuis longtemps à cette visibilité du politique dans les décisions qui entourent le cancer du sein (Ménoret, 2006). La gestion paternaliste de ses dispositifs de prise en charge préventive s'appuie sur un discours volontiers alarmiste du style: «En montrant ses seins, Madame X a sauvé sa vie». L'archaïsme angoissant, le danger de mort, sur lequel fonctionne l'injonction à prévenir le cancer du sein par le truchement de messages qui argumentent sa conviction sur fond d'épidémiologie de mortalité, ne parle en France qu'à $45 \%$ des femmes à qui s'adresse ce discours comminatoire. Les campagnes de prévention en faveur du cancer du sein, selon l'Institut de Veille sanitaire, ont en effet enregistré une augmentation de la participation au dépistage en passant de $33 \%$ en 2003 à $45 \%$ en 2005. Toujours selon Dewey, une extension des règles de la méthode scientifico-politique à l'ensemble de l'humanité en cause plutôt qu'au seul laboratoire de recherche ou cabinet ministériel serait beaucoup plus opérante que ce discours du doute qui n'ose se dire. 
L'incertitude ne concerne d'ailleurs pas que la notion de risque. La promotion de la prévention repose, entre défiance et confiance, sur des objectifs de fait assez ambivalents puisqu'il s'agit non pas de prévenir l'apparition d'un cancer mais de la détecter le plus précocement possible. De fait, ce qu'on appelle prévention dans le domaine du cancer du sein relève au mieux d'une prévention secondaire. En effet, pas de prévention primaire du cancer du sein dans le contexte que l'on vient de décliner. C'est en réalité pour mieux guérir qu'il faut prévenir, c'est-à-dire, en l'état actuel des savoirs, des techniques et des choix de la cancérologie française - si l'on met de côté un dépistage génétique qui radicaliserait la prévention avec une mesure prophylactique d'ablation - dépister. Bien que les pouvoirs publics entretiennent massivement un discours autour de la notion de prévention, c'est donc - basé sur des facteurs de risque élémentaires — de dépistage de cancers avérés dont il s'agit réellement.

L'incertitude est au cœur de la mission curative de la cancérologie. Dans un article intitulé «Breast Cancer: the Good News and the Bad News » publié en juin 1996 dans Atlantic Monthly, David Plotkin écrivait : «L'effet majeur du développement de la mammographie a été de masquer notre incapacité à guérir ce vieux cancer qu'est le cancer du sein en l'enterrant sous les cas de nouveaux cancers que sont les cancers précocement détectés. » Depuis plusieurs décennies, les soins s'organisent sur le même modèle: chirurgie, radiothérapie et chimiothérapie. Tous les cancers ne sont pas guéris tandis que chirurgie et radiothérapie semblent arrivées à saturation de leur potentialité éradicatrice. C'est également dans ce contexte qu'il faut lire l'hégémonie du paradigme de la détection précoce, discrètement mais efficacement, soutenu par les grands laboratoires. La traque du plus petit cancer possible que l'on pourra guérir facilement — quête exacerbée aujourd'hui dans la recherche de gènes de prédisposition au cancer du sein - est une quête d'espoir. Cet espoir est totalement investi dans la science, une science idéale, décisive et lucrative.

\section{RISQUE ET RESPONSABILITÉ INDIVIDUELLE}

Plusieurs recherches ont déjà pensé la construction du risque et son évaluation en essayant de démêler les différents facteurs, notamment sociaux, susceptibles d'intervenir lorsqu'une décision publique est prise à l'égard d'un risque (Brunk 1991; Cranor 1990; Hansson 1993). La détermination d'un risque à partir de méthodes statistiques ne suffit plus à lui attribuer une autorité objective puisque l'on sait désormais le rôle non moins prépondérant des valeurs et des idéologies dans son élaboration et dans sa diffusion. Les jugements de valeur font partie intégrante du risque dans la façon dont il est calculé, communiqué, perçu et in fine accepté.

C'est sur l'une de ces valeurs que l'on va maintenant conclure, laquelle émerge en filigrane à travers l'histoire du risque de cancer du sein : tant celle de son émergence que celle de son traitement. Cette valeur est celle de la responsabilité individuelle. On l'a vu, l'élaboration de la notion de risque en cancérologie a plus souvent reposé sur des facteurs individuels de comportements que sur des approches de groupes qui seraient déterminés par leur exposition involontaire à des facteurs de risques exogènes. Comme 
en attestent, dans les discours français sur la prévention, le tabac abominé et l'amiante ignorée: l'épistémologie du risque de cancer repose en France sur une notion d'environnement réduite à des comportements individuels agrégés. Parallèlement à ce mouvement, la notion de risque a longtemps été mobilisée dans des perspectives beaucoup plus libérales, en l'occurrence en cabinet médical privé, que publiques et populationnelles, c'est-à-dire dans des cabinets ministériels. Cette éthique de l'individuation des responsabilités de prévention, que l'on remarque en observant l'usage de la notion de risque par les experts de santé, ne conçoit pas de mouvement collectif. Le cancer, en France, se pense autour de questions très individualisantes — pourquoi moi? — et fort peu politisées. Comme on a pu le documenter et le montrer in situ (Ménoret, 1999), la cancérologie œuvre, dans l'activité la plus quotidienne et la plus ordinaire de ses services, à produire une expérience esseulée du cancer. Bien peu d'initiatives communautaires ont su émerger de cette épreuve, élaborée à travers le travail médical sur un mode solitaire dominant. On retrouve donc cette composante d'individuation dans le fondement de la valeur de responsabilité individuelle qui est au principe du ciblage des populations de femmes concernées par le dépistage. Ses conséquences sont loin d'être socialement neutres.

En individualisant la responsabilité des femmes à l'égard du cancer du sein, ce dispositif qui prescrit une forme d'autosurveillance, actualise en quelque sorte le «c'est de ta faute» mis au jour par Crawford il y a 30 ans (Crawford, 1977), dans ce qu'il nommait alors une «idéologie de la culpabilisation de la victime». Ce premier type de conséquence affranchit la médecine de toute critique intrinsèque: en mettant l'accent sur la responsabilité personnelle des femmes, les discours de prévention leur attribuent implicitement la cause essentielle, non de leur cancer du sein, mais de son éventuelle incurabilité s'il est détecté trop tard. Compte tenu du taux de mortalité de cette maladie, cette répercussion est conséquente. Un deuxième type de conséquence disculpe, lui, les risques de nature environnementale - aux enjeux économiques souvent majeurs — en les soustrayant tout simplement à l'analyse. Le cancer du sein, en France, n'a jamais été envisagé d'un point de vue écologique, c'est-à-dire comme une pathologie possiblement liée à des causes externes ou à des expositions involontaires. En misant sur la seule responsabilité individuelle, les choix scientifiques et politiques du traitement du risque de cancer du sein en France assurent la promotion d'un seul mode de prévention aux dépens de tout autre option. Privilégier une approche singulière alors qu'une pluralité de facteurs de risque sont considérés — ailleurs qu'en France — en jeu dans l'apparition d'un cancer du sein, c'est incontestablement limiter les options de protection du corps social féminin. Quand l'approche requise repose de surcroît sur une idéologie de la responsabilité personnelle, le principe de responsabilité sociale supposé faire le lit du système de santé français ne semble pas fonctionner au mieux. La prévention du cancer du sein, c'est assurément l'affaire de la cancérologie, de la sénologie et de la radiologie.

Le corps médical, ou plus exactement son élite à laquelle appartiennent assurément la cancérologie, la sénologie ou encore la radiologie, jouit en France d'une 
autonomie qui ne cesse de surprendre, eu égard aux évolutions enregistrées ces dernières décennies par la profession médicale dans la plupart des pays occidentaux (Freidson, 2001; Marks, 1999). On a décliné dans cet article quelques-uns des points remarquables qui ont accompagné le développement, en France, du traitement du risque de cancer du sein : des décisions médico-centrées, sans concertation politique, sans débat public, sans partage des compétences, pas d'administration de la preuve, pas d'évaluation des programmes de dépistage, un discours apologétique en sa faveur, la médicalisation d'un secteur important de santé publique et puis, finalement, pas de prévention stricto sensu du cancer du sein... Concluons avec Peneff $(2005: 15)$ : «Si le corps médical a perdu certaines positions, il a compensé en conquérant la faveur du monde social et politique pour ce qui constitue un nouveau marché: l'idée de santé supplante désormais celle de maladie. » Cette stratégie est de mise depuis déjà fort longtemps avec le risque de cancer du sein.

\section{RÉSUMÉ}

Cet article porte sur l'élaboration du risque de cancer du sein en France et sur ses conséquences sociales. Il s'appuie sur des données historiques et sociologiques pour tracer l'évolution de sa prévention, de la fin du XIX siècle aux tests contemporains de prédisposition génétique. II montre que la détection précoce s'est imposée comme la technique d'excellence de prévention du cancer du sein, dans un monde où les thérapeutiques évoluent peu en matière de guérison, d'une part, et dans lequel les discours alternatifs n'ont eu que très peu d'audience auprès des publics concernés, d'autre part. Basée exclusivement sur le dépistage, l'entreprise française de prévention du cancer du sein a progressivement transformé des personnes en bonne santé en patientes asymptomatiques - dans un processus de médicalisation du risque - et des populations ciblées de femmes en population à risque - dans un processus de naturalisation du même risque. L'entreprise de surveillance qui organise cette situation est l'œuvre d'une autonomie médicale qui légitime un «faute de mieux», en l'absence de remède efficace.

\section{ABSTRACT}

This paper concerns the study of the risk of breast cancer in France and its social consequences. It is based on historical and sociological data tracing the evolution of its prevention from the end of the $19^{\text {th }}$ century to contemporary genetic predisposition tests. It shows that early screening has proved to be the best breast cancer prevention technique in a world where cure-ensuring treatments are not only slow to develop, but in which alternative methods have received little attention from the public most concerned. Based exclusively on screening, breast cancer prevention efforts in France have gradually transformed healthy individuals into asymptomatic patients - in a process of risk medicalization — and targeted groups of women into groups at risk - in a process of naturalization of the same risk. The supervisory body overseeing this situation is the work of an autonomous medical establishment that legitimizes a «lesser evil» in the absence of an effective cure. 


\section{RESUMEN}

Este artículo se refiere a la elaboración del riesgo de cáncer del seno en Francia y sobre sus consecuencias sociales. Se basa en datos históricos y sociológicos para trazar la evolución de su prevención, de finales del siglo XIX a las pruebas contemporáneas de predisposición genética. Pone de manifiesto que la detección precoz se impuso como la técnica de excelencia de prevención del cáncer del seno, en un mundo donde las terapéuticas evolucionan poco en cuanto a curación, por una parte, y en el cual los discursos alternativos tuvieron muy poca audiencia para los públicos interesados, por otra parte. Basada exclusivamente en la detección, la empresa francesa de prevención del cáncer del seno transformó progresivamente personas en buena salud en pacientes asintomáticas - en un proceso de medicalización del riesgo - y poblaciones específicas de mujeres en población de riesgo - en un proceso de naturalización del mismo riesgo - L . La empresa de vigilancia que organiza esta situación está en obra de autonomía médica que legitima el «a falta de algo mejor», en ausencia de remedio eficaz.

\section{BIBLIOGRAPHIE}

Altman, R. (1996), Waking Up Fighting Back, Boston, Little, Brown and Company.

Armstrong, D. (1995), «The rise of surveillance medicine», Sociology of Health and Illness, vol. 17, $\mathrm{n}^{\circ}$ 3, p. 393-404.

Bertillon, J. (1906), De la fréquence des principales causes de décès à Paris pendant la seconde moitié du XIX ${ }^{e}$ siècle, et notamment pendant la période 1886-1905, Paris, Imprimerie municipale.

Boissonnat, V. (2003), «"Un objet-réseau” : la radiographie de dépistage du cancer du sein », in G. Cresson, M. Drulhe et F.-X. Schweyer (sous la dir.), Coopérations, conflits et concurrences dans le système de santé, Rennes, Éditions ENSP, p. 47-73.

Brunk, C.L. (1991), «Is a Scientific Assessment of Risk Possible? Value Assumptions in the Canadian Alachor Controversy", Dialogue, vol. 30, p. 235-247.

Cachin, Y. (1986), La lutte contre le cancer en France. Perspectives-Propositions. Rapport au ministre des Affaires sociales et de la Solidarité nationale et au Secrétaire d'État chargé de la Santé, Paris, La documentation française.

Cassedy, J.H. (1984), American Medicine and Statistical Thinking. 1800-1860, Cambridge, Harvard University Press.

Chateauraynaud F. et D. Torny (1999), Les sombres précurseurs. Une sociologie pragmatique de l'alerte et $d u$ risque, Paris, Éditions de l'EHESS.

Cranor, C.F. (1990), «Some Moral Issues in Risk Assessment», Ethics, vol. 101, p. 123-143.

Crawford, R. (1977), «You Are Dangerous to Your Health : the Ideology and Politics of Victims-Blaming», International Journal of Health Services, vol. 7, $\mathrm{n}^{\circ}$ 4, p. 663-680.

Cuyler Hammond E. et D. Horn (1958), «Smoking and Death Rates. Report on Forty-Four Months Follow-up of 187783 Men. II. Death rates by cause», Journal of the American Medical Association, vol. 166, p. 1294-1308.

DAB, W. (2006), Préface à G. Welch, Dois-je me faire tester pour le cancer? Peut-être pas et voici pourquoi, Sainte-Foy, Presses de l'université Laval.

Dewey, J. (1954 [1927]), Public and Its Problems, Chicago, Swallow Press.

Doll, R. et R. Рето (1976), «Mortality in Relation to Smoking: 20 Years' Observations on Male British Doctors», British Medical Journal, vol. 2, p. 1525-1536.

Fagnani, F. et al. (1985), «Enquête nationale sur le radiodiagnostic», Journal de Radiologie, vol. 86, n² 2, p. $167-174$.

Fagot-Largeault, A. (1989), Les causes de la mort. Histoire naturelle et facteurs de risque, Paris, Vrin. 
Fisher, J.P. et al. (1998), «Tamoxifen for Prevention of Breast Cancer: Report of the National Surgical Adjuvant Breast and Bowel Project P-1 Study», Journal of the National Cancer Institute, vol. 90, p. 1371-1388.

Fisher, R.A. (1957), «Dangers of Cigarette-Smoking », British Journal of Medicine, I, p. 297-298 et II, p. 43. Fosket, J. (2004), «Constructing “High-Risk Women” : The Development and Standardization of a Breast Cancer Risk Assessment Tool», Science, Technology, and Human Values, vol. 29, n 3, p. 291-313.

Fox, R. (1980), «The Evolution of Medical Uncertainty», Health and Society, vol. 58, n 1, p. 1-49.

Freidson, E. (2001), Professionalism. The Third Logic, Chicago, University of Chicago Press.

Freidson, E. (1984 [1970]), La profession médicale, Paris, Payot.

Hacking, I. (1990), The Taming of Chance, Cambridge, Cambridge University Press.

Hacking, I. (1975), The Emergence of Probability, Cambridge, Cambridge University Press.

Hansson S.O. (1993), «The False Promises of Risk Analysis», Ratio, VI, p. 16-26.

Junod, B. et R. MAssé (2003), «Dépistage du cancer du sein et médicalisation de la santé publique », Santé publique, vol. $15, \mathrm{n}^{\circ} 2$, p.125-129.

Kasper, A. et S. Ferguson (2000), Breast Cancer, Society Shapes an Epidemic, New York, St Martin Press.

Kaufert, P. (1998), «Women, Resistance, and the Breast Cancer Movement», in Lock, M. et P. Kaufert (éds), Pragmatic Women and Body Politics, Cambridge, Cambridge University Press, p. 287-309.

Kevles, B. (1997), Naked to the Bone: Medical Imaging in the Twentieth Century, Helix Books, AddisonWesley.

LE GALÈs, C. et al. (1990), «Le dépistage mammographique du cancer du sein: explosion des pratiques, multiplication des programmes », Bulletin épidémiologique hebdomadaire, vol. 1, nº 3, p. 3-4.

Lefaure, C. et al. (1990). «Le dépistage mammographique du cancer du sein », Le Concours Médical, vol. 113, $\mathrm{n}^{\circ} 33$, p. 2991-2993.

Leopold, E. (1999), A Darker Ribbon, Boston, Beacon Press.

Lerner, B. (2001), The Breast Cancer Wars, New York, Oxford University Press.

Lickint, F. (1935), « Der bronchialkrebs der raucher», München med wochenschr, n ${ }^{82}$, p. 1232-1234.

Lock, M. et P. Kaufert (1998), Pragmatic Women and Body Politics, Cambridge University Press.

Love, S. (1990) Dr. Susan Love's Breast Book, Reading, Addison-Wesley.

Marks, H. (1999), La médecine des preuves. Histoire et anthropologie des essais cliniques. Paris, Synthélabo, coll. «Les Empêcheurs de penser en rond».

Ménoret, M. (2006), «Prévention du cancer du sein : cachez ce politique que je ne saurais voir », Nouvelles questions féministes, vol. 26, $\mathrm{n}^{\circ} 2, \mathrm{p} .32-47$.

Ménoret, M. (2001), «Genesis of the Notion of Stage in Oncology: the French 'Enquête Permanente Cancer' (1943-1952)», Social History of Medicine, vol. 15, n 2, p. 291-302.

Ménoret, M. (1999), Les temps du cancer, Paris, Éditions du CNRS (nouvelle édition augmentée en 2007), Paris, Le bord de l'eau.

Montini, T. et S. RuzeK (1989), «Overturning Orthodoxy: the Emergence of Breast Cancer Treatment Policy ", Research in the Sociology of Health Care, p. 3-32, Greenwich, JAI Press.

Paicheler, G. (2002), Prévention du sida et agenda politique. Les campagnes en direction du grand public (1987-1996), Paris, Éditions du CNRS.

Paulsen, M. (1994), «The Cancer Business», Mother Jones, n 4, p. 23-24.

Peneff, J. (2005), La France malade de ses médecins, Paris, Les Empêcheurs de penser en rond.

Pinell, P. (1992), Naissance d'un fléau. Histoire de la lutte contre le cancer en France (1890-1940), Paris, Métailié.

Poтts, L.K. (2000), Ideologies of Breast Cancer. Feminist Perspectives, New York, St. Martin Press.

Proctor, R. (1995), Cancer Wars, New York, Basic books.

Rosser Matthews, J. (1995), Quantification and the Quest for Medical Certainty, Princeton, Princeton University Press.

Schwartz, D. (1994), Le jeu de la science et du hasard. La statistique et le vivant, Paris, Flammarion.

Simpson, C. (2000), «Controversies in Breast Cancer Prevention», in Potts (2000), p. 131-152. 
Stabiner, K. (1997), To Dance with the Devil, New York, Delta.

TABAR, L. et al. (1985), «Reducing in Mortality from Breast Cancer After a Mass Screening with Mammography", Lancet, I, p. 829-832.

WaIt, S. et al. (1997), «Dépistage individuel du cancer du sein en France », Bulletin du Cancer, vol. 84, n 6, p. 619-624.

WeLCh, G. (2005), «Search and Destroy — The Right Cancer Strategy for Europeans?», European Journal of Cancer, vol. 41, p. 660-663.

Yerushalmy J. et E. Palmer Caroll (1959), «On the Methodology of Investigations of Etiologic Factors in Chronic Diseases", Journal of Chronic Disease, 10, p. 27-40. 